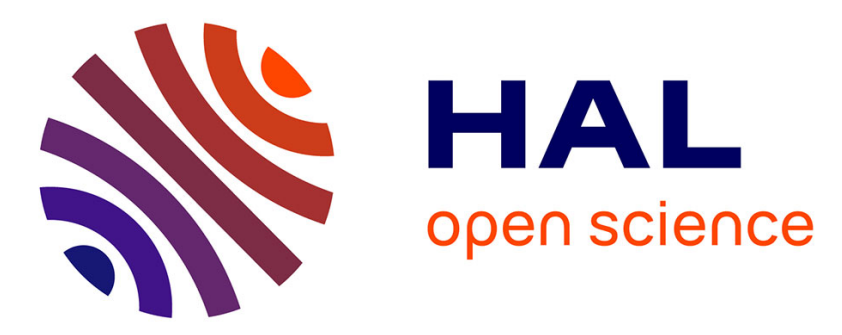

\title{
Taphonomie et diagnose sexuelle primaire à partir de l'os coxal: du terrain au laboratoire
}

\author{
Cindy Dentz, Bruno Bizot, Anne Richier, Aurore Schmitt
}

\section{To cite this version:}

Cindy Dentz, Bruno Bizot, Anne Richier, Aurore Schmitt. Taphonomie et diagnose sexuelle primaire à partir de l'os coxal: du terrain au laboratoire. Bulletins et Mémoires de la Société d'anthropologie de Paris, 2020, 32 (3-4), pp.158-167. 10.3166/bmsap-2020-0094 . hal-02991046

\section{HAL Id: hal-02991046 \\ https://hal.science/hal-02991046}

Submitted on 5 Nov 2020

HAL is a multi-disciplinary open access archive for the deposit and dissemination of scientific research documents, whether they are published or not. The documents may come from teaching and research institutions in France or abroad, or from public or private research centers.
L'archive ouverte pluridisciplinaire HAL, est destinée au dépôt et à la diffusion de documents scientifiques de niveau recherche, publiés ou non, émanant des établissements d'enseignement et de recherche français ou étrangers, des laboratoires publics ou privés. 
Taphonomie et diagnose sexuelle primaire à partir de l'os coxal : du terrain au laboratoire

Taphonomic processes and primary sexual determination from the os coxae: from the field to the laboratory

Dentz C., Bizot B., Richier A., Schmitt A 
Résumé: Une grande partie des analyses ostéologiques reposent sur la détermination du sexe de l'individu étudié. Les méthodes basées sur l'os coxal- dont le dimorphisme est commun à toute l'espèce humaine- permettent d'obtenir des résultats très probants. Cependant, la structure de l'os coxal, principalement composée d'os spongieux, le rend fragile. Ainsi, comme de précédentes études l'ont démontré, on observe une conservation différentielle des parties qui le composent liées à des raisons taphonomiques mais également aux manipulations du terrain au laboratoire. Cette étude s'est appuyée sur 302 individus issus de la fouille du cimetière médiéval et moderne de La Ciotat, sélectionnés en fonction de deux critères : la présence d'au moins un élément de l'os coxal et le fait qu'ils aient été lavés au préalable. Elle a permis de confirmer la faible conservation de l'os coxal. De plus, l'application des méthodes de détermination du sexe morphoscopique et de la DSP a mis en évidence l'absence de parties spécifiques, plus fragiles. Nous avons également testé la corrélation entre le sexe estimé de l'individu et l'état de conservation de l'os coxal. La prise de données directement lors de la phase terrain s'avère une étape incontournable pour garantir la diagnose sexuelle primaire du maximum d'individus.

Mots clés : os coxal, diagnose sexuelle, conservation, terrain, laboratoire.

Abstract: Much of osteological analysis focuses on the sex determination of the individual being studied. Because sexual dimorphism of the os coxae is shared by all Homo sapiens sapiens, analysis of it lends itself to convincing determinations of sex. Nevertheless, apart from its reliability, its structure, which is mainly composed of cancellous bone, makes it fragile. Thus, as previous studies have shown, there is differential preservation of the different parts of the os coxae, but also as a consequence of their handling from the time of excavation to laboratory study. This study was based on 301 individuals from the excavation of the medieval and modern cemetery of La Ciotat, previously selected based on two criteria: the presence of at least one of the two ossa coxae, and the fact that they had been washed beforehand. The Anatomical Preservation Index (API) ICA established by S. Bello confirmed the poor preservation of the ossa coxae. Moreover, the use of morphological sex determination and DSP (Diagnose Sexuelle Probabiliste) methods revealed the frequent absence of the most fragile parts of the element.. A correlation between sex and preservation was also performed. Finally, we highlight the the importance of data and observations collected during excavation, owing to a significant loss of information that occurs between field excavation and laboratory study.

Key words os coxae, sex assessment, preservation, field recovery, laboratory analysis. 


\section{Introduction}

La détermination du sexe est une étape cruciale de l'analyse d'une série ostéologique, qu'il s'agisse de discuter de pratiques mortuaires, de recrutement, d'état sanitaire, etc. Il est admis, depuis plusieurs décennies, que l'os coxal, qui exprime à lui seul la quasi-totalité du dimorphisme sexuel du bassin osseux, est l'élément le plus pertinent pour une diagnose sexuelle fiable dans la mesure où ce dimorphisme est commun à toute l'espèce humaine et pas seulement à une population en particulier [1]. En France, deux méthodes sont utilisées en routine, notamment sur le matériel anthropologique issu de fouilles archéologiques : la méthode morphoscopique développée par J. Bruzek [2] et la Diagnose Sexuelle Probabiliste [3,4]. Toutefois, ces méthodes nécessitent une bonne conservation de l'os coxal pour garantir la fiabilité de la diagnose.

Or, cet élément osseux est fragile et sa préservation variable d'un site à l'autre [5]. En effet, le rapport entre os compact et os spongieux n'est pas favorable à sa conservation [6]. Les parties les plus sensibles aux dégradations taphonomiques sont les extrémités de l'aile iliaque, le corps du pubis et l'épine sciatique $[7,8,9]$, autant d'éléments entrant dans les critères pris en compte dans les méthodes sus-citées. A titre d'illustration, sur 860 os coxaux issus de caveaux d'époques médiévale et moderne de Grenoble Saint-Laurent, 533 n’ont pu être exploités [10].

Pour pallier cette limite, la diagnose sexuelle secondaire [5] qui permet d'approcher avec une marge d'erreur connue la détermination du sexe de sujets ne présentant pas d'os coxal a été proposée. Les fonctions discriminantes établies sont propres à la série ostéologique étudiée et tiennent compte, par conséquent, de ses spécificités. Cependant, pour paramétrer ces fonctions, il est nécessaire qu'un nombre suffisant de sujets féminins et masculins ait bénéficié d'une diagnose primaire à partir de l'os coxal. 
Afin d'augmenter les chances de déterminer le sexe d'un sujet exhumé sur une fouille archéologique, nous sommes nombreux à avoir envisagé d'effectuer la détermination sexuelle sur le terrain. Une première expérience de ce type a été tentée par M. Guillon [11] sur le cimetière médiéval de Tournedos à Porte-Joie (Eure, Normandie) portant sur 751 individus. Le choix de procéder sur le terrain à la diagnose sexuelle sur les os coxaux apparaissant les plus fragiles a été fait sur la fouille d'un second cimetière médiéval de cette même commune ; les résultats de cette expérience ont été publiés par W. Berthon et al. [12]. Malheureusement, il était trop tard pour que leurs conclusions puissent être mises à profit lors de la fouille préventive du cimetière SaintJacques à la Ciotat $[13,14]$ dont il sera question ici.

Dans le cadre de cette opération archéologique, la détermination du sexe, in situ, sur les os coxaux en appliquant la méthode morphoscopique [2] a été prescrite dans le cahier des charges auquel devait répondre l'opérateur. Cette prise de données en cours de fouille a été envisagée comme une première information, au même titre que l'évaluation de la classe d'âge des défunts consignée dans les fiches de terrain en vue d'obtenir une esquisse du recrutement dans les zones fouillées, l'un des enjeux étant la localisation d'une aire d'ensevelissement réservée à l'hôpital contigu du cimetière. En outre, il avait été prévu d'emblée que l'étude anthropologique postfouille ne pourrait porter que sur un échantillon de 400 individus afin de dégager des pistes de réflexion sur le contexte funéraire et orienter d'autres études anthropologiques.

La préparation de la collection anthropologique issue des fouilles de la Ciotat se poursuivant, plusieurs travaux universitaires ont été engagés ${ }^{1}$. Dans l'un d'eux, la détermination sexuelle d'un échantillon de 302 individus adultes pourvus d'un bassin a été effectuée en appliquant les méthodes morphoscopique et probabiliste en prévision d'une diagnose sexuelle secondaire. Cette

\footnotetext{
${ }^{1}$ Notamment les travaux doctoraux de M. Perrin [15]
} 
nouvelle approche offrait l'opportunité de quantifier la perte d'informations entre la phase terrain et l'étude en laboratoire, de comparer les résultats obtenus et d'évaluer l'impact des dégradations subies selon le sexe. Bien que les protocoles appliqués sur le terrain diffèrent sensiblement (pas de DSP in situ), il nous a semblé que les enjeux en termes de résultats et de moyens à déployer sur les fouilles archéologiques sont suffisamment importants pour rendre compte à notre tour de cette expérience et la confronter à celle de W. Berthon et ses collègues [12].

\section{Matériel}

L’opération archéologique du carré Saint-Jacques à la Ciotat a été réalisée en 2009. Elle a permis d'exhumer sur un peu plus de 1000 m², 1245 individus (933 adultes et 312 immatures) en dépôt primaire. Ce cimetière enclos attenant à l'hôpital Saint-Jacques a été créé ex nihilo en 1581 et abandonné en 1831. Les défunts y étaient inhumés dans des fosses ou dans des cercueils disposés en rangées plus ou moins bien organisées. Trois phases d'occupation séparées par des remblais ont pu être distinguées, ce qui permet d'inscrire chaque sépulture dans une période précise.

Les recoupements entre sépultures sont nombreux et les squelettes complets sont rares. De fait, une évaluation de la représentation globale de chaque sujet a mis en évidence que 243 individus étaient complets ou sub-complets, 505 moyennement représenté (par au moins la moitié de ses ossements) et 497 incomplets (par moins de la moitié de ses ossements), ceux-ci étant représentés, en réalité, par quelques os ou esquilles [13]. Au final, 529 individus adultes ou de taille adulte possédaient au moment de la fouille au moins un os coxal.

L'étude anthropologique préliminaire réalisée sur un échantillon de 400 individus dans le cadre de la post-fouille de l'opération a permis d'examiner 324 adultes ou grands adolescents. Parmi ceux-ci, 224 étaient pourvus d'au moins un os coxal au moment de la fouille et une diagnose sexuelle a pu être réalisée sur le terrain sur 203 d'entre eux (90\%). En post-fouille, 131 (58\%) des 
sujets en question ont pu de nouveau être déterminés avec la méthode morphoscopique et seulement 49 (22\%) ont pu bénéficier d'une DSP. Aucun désaccord n'a été relevé par les auteurs de l'étude ${ }^{2}$ entre le terrain et le laboratoire pour la méthode morphoscopique. En revanche, dans 9 cas (18\%), la détermination probabiliste s'est avérée en contradiction avec ces résultats.

Dans le cadre de notre étude, nous avons retenu 302 individus parmi les 529 adultes en position primaire que contenait notre collection. Notre sélection de départ était basée sur trois critères simples, les squelettes devaient avoir été au moins lavés, posséder au moins un élément d'os coxal et appartenir aux classes d'âge adultes et grands immatures. Cependant, le sexage des grands immatures n'a pas été possible en raison de leur fusion inachevée. C'est pourquoi notre échantillon d'étude ne contient que des individus adultes.

\section{Méthodes}

Dans un premier temps, nous avons travaillé à l'échelle de l'os coxal $(\mathrm{N}=604)$. Afin d'évaluer l'état de ces os après lavage et conditionnement, nous avons appliqué l'Indice de Conservation Anatomique [16, 17] appréciant la conservation de l'os selon 6 stades auquel a été ajouté un septième stade « Non Identifiable » réservé aux pièces trop fragmentées pour permettre une telle évaluation (tableau 1, figure 1).

La méthode morphoscopique [2] est basée sur l'observation de cinq caractères morphologiques (surface pré-auriculaire, grande échancrure ischiatique, arc composé, bord inférieur du pubis et rapport ischio-pubien) composés pour certains d'entre eux de sous-critères. Comme l'a mentionné J. Bruzek [2], le nombre de scores corrects obtenus varie sensiblement en fonction du sexe et de l'échantillon de référence pris en compte. Par ailleurs, l'état de conservation des os

\footnotetext{
${ }^{2}$ Catherine Rigeade, Renaud Lisfranc et Anne Richier [13]
} 
conduit souvent à privilégier le segment sacro-iliaque, ce qui est moins pénalisant que s'il s'était agi du complexe ischio-pubien où le nombre d'erreurs ou indéterminé est important, au point que l'écart entre le nombre de déterminations correctes obtenues pour chacun des sexes, les deux échantillons de référence réunis, s'avère statistiquement significatif (80\% de réussite pour les sujets d'une morphologie masculine et $93 \%$ pour les sujets d'une morphologie féminine, $\mathrm{p}$ 0.00012 ; d'après les données de Bruzek [2], table 4 série B).

Au moment de la fouille, seule la méthode morphoscopique a été appliquée lors du démontage des squelettes, la mise en œuvre de la DSP sur le terrain ayant été jugée trop complexe. Seuls les résultats obtenus (masculin, féminin, indéterminé) ont été reportés sur la fiche de sépulture par les quatre archéo-anthropologues en charge de l'enregistrement sur le terrain ${ }^{3}$ ce qui ne permet pas d'évaluer a postériori comment les différents critères de cette méthode ont été pris en compte ou d'apprécier l'érosion de chacun des segments anatomiques en question. Des fiches de conservations ont également été remplies a posteriori à partir de données manuscrites prises lors de la fouille. Malgré cela, compte tenu de l'état de conservation général du matériel anthropologique, il peut être avancé sans risque que, dans bien des cas, seul le complexe sacroiliaque a dû être pris en compte.

En laboratoire, en revanche, afin d'évaluer la possibilité d'appliquer la méthode de J. Bruzek selon l'état de conservation de l'os coxal, nous avons calculé dans combien de cas une attribution sexuelle par caractère était possible. Celle-ci se décline selon trois modalités : sujets d'une morphologie masculin, sujets d'une morphologie féminine, et indéterminé. Nous avons distingué également trois modalités de non attribution à une forme sexuelle : caractère absent, non observable, non identifiable (tableau 2).

\footnotetext{
${ }^{3}$ Gwenhaël Georget, Julie Hernot, Renaud Lisfranc, Catherine Rigeade.
} 
La Diagnose Sexuelle Probabiliste [3] repose sur la prise de 10 mesures. Nous avons calculé pour chacune d'elles le nombre de fois où cet enregistrement était possible sur les 604 os coxaux $^{4}$ de notre échantillon et, par la suite, comparé les fréquences en fonction du sexe en ne tenant compte que des os coxaux qui ont pu bénéficier d'une diagnose sexuelle.

Un de nos objectifs consistait également à évaluer si la dégradation de l'os coxal est sexedépendante ce qui peut, le cas échéant, introduire un biais dans les études de sexe ratio. Une fois la diagnose sexuelle effectuée sur chaque os coxal exploitable, que le sexe soit donné par la méthode morphoscopique ou métrique, nous avons vérifié par un test exact de Fisher si les valeurs obtenues pour chaque stade d'ICA par sexe s'avéraient significativement différentes. Nous avons également comparé les mesures exploitables en fonction du sexe. Par ailleurs, nous avons calculé la fréquence des caractères morphoscopiques présents par sexe et par côté et appliqué un test exact de Fisher afin de savoir si le sexe de l'individu joue sur la conservation de certaines parties anatomiques.

Dans un second temps, nous avons changé d'échelle pour travailler à celle de l'individu $(\mathrm{n}=302)$ afin d'évaluer l'impact des dégradations osseuses qui se produisent entre la phase de terrain et les études menées en laboratoire. Nous avons comparé, par un tableau de contingence, les résultats des diagnoses sexuelles effectuées à chacune de ces phases d'étude et calculé les pourcentages de concordance.

\footnotetext{
${ }^{4}$ Il s'agit du nombre total de coxaux pris en compte dans l'étude. Cet effectif inclut les os coxaux au stade de conservation 0 .
} 


\section{Résultats}

\section{A l'échelle de l'os coxal}

Sur la totalité de notre série (604 os coxaux), les stades de l'ICA cotés après lavage et, si nécessaire, consolidation et reconditionnement des os, indiquent que $51.33 \%$ seulement des os coxaux observés sont représentés par au moins $50 \%$ de l'os (figure 2 ), ce que $\mathrm{S}$. Bello considère comme «bien conservé ». D'ailleurs, le stade 7 qui correspond à un os coxal complet se démarque de façon flagrante des autres valeurs. Les os coxaux dont la conservation n'a pas pu être évaluée (stade 1) ne représentent, quant à eux, que 3,6 \% de l'échantillon.

Par os coxal, quel que soit le côté considéré, l'attribution d'une forme sexuelle (féminine, masculine, ou indéterminée) à chacun des critères de la méthode morphoscopique est rarement possible pour la margo inferior ossis pubis et pour le rapport ischium/pubis. En effet, il s'agit d'une région particulièrement fragile en raison de la finesse de la branche pubienne. De même, l'évaluation de la forme de la grande incisure ischiatique requiert la conservation de presque la totalité de la longueur de l'os et, l'arc composé, la conservation de l'ilium. Or, la mauvaise conservation de ces éléments avait déjà été mise en évidence par Sauter et Privat [18]. Globalement, l'attribution à une forme sexuelle est possible dans moins de $50 \%$ des cas quel que soit le critère observé (figure 3) ce qui explique que de nombreux adultes n'aient pas bénéficié d'une diagnose sexuelle.

Nous pouvons constater de fortes disparités dans le nombre de mesures de la DSP qu'il a été possible de prendre sur notre échantillon (figure 4). Sans grande surprise, la fréquence des mesures se basant sur la partie ischio-pubienne est basse (PUM, SPU, ISMM), de même que les mesures qui requièrent une conservation complète de la crête iliaque (DCOX, SCOX). En revanche, les mesures appartenant à la zone sacro-iliaque et au sourcil acétabulaire ont pu être 
fréquemment enregistrées (IIMT, SS, SA, SIS, VEAC). La largeur est moins fréquemment mesurable car les bords latéraux et médiaux de la crête iliaque sont plus fragiles que son sommet et l'ischium pris en compte dans les mesures de longueur est une partie plutôt résistante.

\section{La conservation des os coxaux en fonction du sexe}

Si l'on tient compte des os coxaux pour lesquels le sexe a pu être attribué $(n=319)$, le test non paramétrique de Fisher ne révèle pas de différence de conservation entre les os coxaux attribués au sexe masculin ou féminin, même pour le stade de conservation 6 (figure 5) où l'on compte deux fois plus du sexe masculin ou féminin.

Concernant la méthode de Diagnose sexuelle Probabiliste [3], il n'y a pas non plus de différences statistiquement significatives entre la fréquence des mesures prises sur les os coxaux féminins et masculins (figure 6), mais toutes les valeurs féminines sont inférieures aux valeurs masculines comme l'avait mentionné S. Bello dans sa thèse [17].

En revanche, pour la méthode morphoscopique, la présence des critères anatomiques exploitables apparaît, dans notre échantillon, significativement différente entre les deux sexes pour l'arc composé du côté gauche et la margo inferior pour l'os coxal droit (tableau 3).

\section{A l'échelle de l'individu}

Sur notre corpus de 302 individus, 275 individus (91\%) ont bénéficié d'une diagnose sexuelle sur le terrain avec la méthode morphoscopique, pour seulement $168(56 \%)$ en laboratoire quelle que soit la méthode, ce qui représente une perte de 35\% d'informations entre le terrain et le laboratoire. Les nombreuses manipulations ayant eu lieu entre le terrain et la reprise de l'étude ont par conséquent entraîné une érosion importante des parties anatomiques déterminantes pour 
la diagnose sexuelle (sont inclus ici les individus de sexe indéterminé car ils ont bénéficié d'une diagnose sexuelle).

Nous avons pu comparer la concordance des sexes attribués sur le terrain et a posteriori par la méthode morphoscopique et la DSP. Il s'avère que 21,5\% des déterminations sont en désaccord (tableau 4). Par ailleurs, la diagnose sur le terrain conduit à classer 9 sujets en indéterminé alors que ceux-ci ont pu être attribués à l'un des deux sexes en laboratoire.

Pour 121 individus (40\%), la DSP a pu être appliquée sur au moins un os coxal, tandis que cinq d'entre eux seulement n'ont pas bénéficié de la méthode morphoscopique (non observable). Enfin, quelle que soit la méthode, le sexe a pu être déterminé sur 173 individus (57.4\%).

\section{Discussion}

Notre étude confirme le problème de la conservation différentielle des différentes parties constituant l'os coxal, mais, à ces altérations découlant de la constitution de l'os, il faut ajouter celles de nos pratiques. Nous avons noté que du terrain au laboratoire les os coxaux ont subi de sévères pertes de substance osseuse, ce qui réduit inévitablement le nombre d'individus pouvant bénéficier d'une diagnose sexuelle primaire. Les multiples manipulations (dégagement, prélèvement, conditionnement, transport, lavage puis reconditionnement) sont largement responsables de ce résultat. Ce constat a déjà été établi et commenté notamment dans le cas d'études paléopathologiques [19, 20, 21].

On peut également noter au passage que l'Indice de Conservation Anatomique [16,17] apporte une appréciation globale ne garantissant en rien la «mesurabilité » de l'os. Au niveau de l'enjeu - obtenir au moins quatre variables clé de la DSP - cet indice n'apparait pas suffisant pour évaluer les potentialités d'une série. Pour pallier cette limite, une attention particulière portée à 
l'os coxal sur le schéma de conservation, serait souhaitable. L'os coxal devrait être représenté par les trois parties anatomiques détachées les unes des autres (ilium, ischium, pubis) afin de permettre une cotation précise des zones conservées pour chaque élément. Une autre solution plus directe et produisant une donnée viable et durable est d'appliquer systématiquement la DSP sur le terrain.

La perte irrémédiable de 35\% d'informations au cours de ces différentes manipulations est constatée sur le site de la Ciotat est plus importante qu'à Porte-Joie (Eure, Normandie) où les os coxaux semblent plus résistants. Pour ces raisons au moins, on ne peut que souscrire à l'application des méthodes de détermination du sexe sur l'os coxal dès la phase de terrain [12]. Encore faut-il que les informations collectées soient de qualité comparable. Les mauvaises concordances entre le terrain et le laboratoire semblent dans les deux cas avoir la même origine : le fait de s'affranchir, sur le terrain, de reporter les cotations conduit au non-respect du principe de majorité qui garantit la fiabilité de la méthode morphoscopique.

Concernant les mesures impliquées dans la DSP, il est intéressant de noter que, hormis IIMT, les distances les plus discriminantes sont celles qui sont le moins souvent mesurable, ce qui explique en partie que l'attribution à un sexe n'est pas toujours possible. La perte de la branche pubienne notamment diminue considérablement les chances de succès. On se retrouve alors avec le corps de l'os qui permet les mesures SIS, SA, SCOX, VEAC, SPU, SS.

Par ailleurs, il a été établi dans de précédentes études [8, 17] que l'os coxal d'un individu masculin est plus robuste que celui d'un individu féminin. Cette robustesse s'apprécie particulièrement dans la zone acétabulaire. De plus, cette zone offre des résultats probants - bien que moins fiables que certaines autres parties plus fragiles de l'os coxal - et se conserve bien. Des mesures impliquant cette zone mériteraient une attention particulière. 
Notre étude a également montré que la variabilité de la conservation de l'os coxal pourrait être liée, en partie, au sexe de l'individu. Toutefois, les différences identifiées pour deux variables (arc composé et margo inferior ossis pubis) ne s'observent que d'un seul côté. Dans la mesure où les défunts sont inhumés sur le dos, on ne peut imputer ce biais aux pratiques funéraires, ce qui nous oriente plutôt vers un biais propre à l'échantillon. Cette observation mériterait d'être vérifiée par une étude tenant compte de l'âge des individus, l'ostéoporose ayant forcément un impact sur la conservation de l'os coxal chez les sujets féminins.

\section{Conclusion}

La détermination du sexe d'un individu conditionne la plupart des études menées sur une série ostéologique, qu'il s'agisse des pratiques mortuaires ou de l'approche anthropo-biologique. Bien que plusieurs méthodes permettent actuellement une diagnose sexuelle fiable, la fragilité intrinsèque de l'os coxal limite fréquemment le nombre d'individus dont le sexe est déterminé. La perte de substance osseuse et la fragmentation au cours des différentes étapes du processus de fouilles archéologiques et du traitement du matériel s'ajoutent aux altérations taphonomiques et anthropiques antérieures.

A contrario de ce que nous avions envisagé lors de la programmation de la fouille de la Ciotat, la méthode morphoscopique introduit des aléas liés à la lisibilité de l'os sur le terrain et à la saisie de nombreuses cotes. Il semble par conséquent préférable de privilégier la DSP. Pour peu que les critères et mesures soient précisément consignés, l'effort consenti sur le terrain nous paraît largement compensé par le gain d'informations obtenues. Et, dès lors que chacun des paramètres entrant dans la détermination du sexe a été consigné, il ne nous paraît pas nécessaire de revenir 
sur ces déterminations dans le cadre de la post-fouille sauf, bien entendu, lorsque la restauration d'une pièce osseuse permet de prendre de nouvelles mesures.

Notre étude, portant sur un nombre plus important de sujets que celle de W. Berthon et ses collaborateurs [12], et n'intégrant pas seulement les os coxaux les plus fragiles, vient renforcer leur constat et nous ne pouvons que souscrire à leurs propositions. Au vu de cette convergence, la détermination du sexe in situ apparaît la meilleure solution. Les deux études incitent donc à prescrire dans le cahier des charges des opérations archéologiques, la réalisation systématique de la diagnose sexuelle primaire sur le terrain en privilégiant la DSP lorsque son application est possible.

\section{Références}

1. Bruzek J, Schmitt A, Murail P (2005) Identification biologique individuelle en paléoanthropologie. Détermination du sexe et de l'âge au décès à partir du squelette. In: Dutour O, Hublin JJ, Vandermeersch B (ed) Objets et Méthodes en Paléoanthropologie, CTHS, Paris, pp 217-245

2. Bruzek $\mathbf{J}$ (2002) A method for visual determination of sex, using the human hip bone. Am $\mathbf{J}$ Phys Anthropol $117: 157-168$

3. Murail P, Bruzek J, Houët F et al (2005) DSP: A tool for probabilistic sex diagnosis using worldwide variability in hip-bone measurements. Bull Mem Soc Anthropol Paris 17 (3-4) : 167-176

4. Bruzek J, Santos F, Dutailly B et al (2017) Validation and reliability of the sex estimation of the human os coxae using freely available DSP2 software for bioarchaeology and forensic anthropology, Am J Phys Anthropol 164: 440-449

5. Murail P, Bruzek J, Braga J (1999) A New Approach to Sexual Diagnosis in Past Populations. Practical Adjustments from Van Vark's Procedure. Int J Osteoarchaeol 9: 39-53

6. Denys C, Patou-Mathis M (2014) Manuel de la taphonomie, Errance, Arles, 7-26, 271p

7. Waldron $\mathrm{T}$ (1987) The relative survival of the human skeleton: implication for palaeopathology. In: Boddington A, Garland AN, Janaway RC (ed), Death, Decay and Reconstruction, Manchester, Manchester University Press, pp 55-64

8. Gaillard J (1960) Détermination sexuelle d'un os coxal fragmentaire. Bull Mem Soc Anthropol Paris, 11 (1-2), pp 255-267

9. Walker PL (1995) Problems of preservation and sexism in sexing: some lessons from historical collections for paleodemographers. In: Saunders SR, Herring A (ed) Grave 
reflections: portraying the past through cemetery studies, Canadian Scholar's Press: Toronto, pp 31-47

10. Hervieu P (2010) Gestion funéraire et paléobiologique de sépulture collective : 1'exemple de caveau au Moyen Age et à l'époque moderne l'exemple des caveaux. Thèse de Doctorat d'Anthropologie biologique. Université de la Méditerranée, Aix-Marseille II

11. Guillon M (1997) Anthropologie de terrain et paléodémographie : études méthodologiques sur les grands ensembles funéraires: applications au cimetière médiéval de TournedosPortejoie (Eure). Thèse de Doctorat en Anthropologie Biologique, Université de Bordeaux I

12. Berthon W, Thomas A, Thomann A et al (2015) Faut-il mener une diagnose sexuelle in situ dans les grands ensembles funéraires ? Le cas du cimetière médiéval de Val-de-Reuil (Eure), «Le Chemin aux Errants ». Bull Mem Soc Anthropol Paris 27 (1-2), pp 26-41

13. Richier A (2011), Thuaudet O, Wicha S (collab). L'îlot Saint-Jacques, du vignoble champêtre au cimetière paroissial. Rapport Final d'Opération de Fouille. Inrap Bouches-du-Rhône, Vol 1

14. Richier A, Bizot B (2018) L'opération archéologique du cimetière de La Ciotat (Bouches-duRhône) : réussites et écueils. In: Carré F, Hincker V, Chapelain de Seréville-Niel C (ed), Rencontre autour des enjeux de la fouille des grands ensembles sépulcraux médiévaux, modernes et contemporains. Publication du Gaaf , 7 : 145-154

15. Perrin M (2019) Etat sanitaire entre ancien régime et révolution industrielle : étude paléoépidémiologique de deux populations provençales, interactions bio-culturelles. Thèse de Doctorat en Anthropologie Biologique Aix- Marseille Université, 550 p

16. Bello S, Signoli M, Rabino M et al (2002) Les processus de conservation différentielle du squelette des individus immatures : implications sur les reconstitutions paléodémographiques. Bull Mem Soc Anthropol Paris 14 (3-4) : 245-262

17. Bello S (2001) Taphonomie des restes osseux humains, Effet des processus de conservation $\mathrm{du}$ squelette sur les paramètres anthropologiques, Thèse de Doctorat en Anthropologie Biologique, Marseille, Aix- Marseille Université et Università degli Studi di Torino

18. Sauter MR, Privat F (1952) Une nouvelle méthode de détermination sexuelle de l'os coxal : l'indice cotylo-sciatique. In: Bull Soc Suisse Anthropol Ethno, 28 : 12-13

19. Ardagna Y, Fort A, Sperendio E (2017) Metric data in biological anthropology: History and handling damage/Les données métriques en anthropologie biologique : entre histoire et endommagement. Bull Mem Soc Anthropol Paris 29 (3-4) : 177-184.

20. Caffell A, Roberts C, Janaway R et al (2001) Pressures on osteological collections: The importance of damage limitation. In Williams E (ed) Human remains: conservation, retrieval and analysis: Proceedings of a conference held in Williamsburg, VA, Nov 7-11th 1999, Archaeopress, Oxford, pp 187-197

21. Caffell A, Roberts C, Janaway R et al (2001) Human skeletal collections: the responsibilities of project managers, physical anthropologists and conservators, and the need for standardized condition assessment, Williams E (ed). In: Human remains: conservation, retrieval and analysis: Proceedings of a conference held in Williamsburg, VA, Nov 7-11th 1999, Archaeopress, Oxford, pp. 199-208 


\section{Légendes}

Tableau 1 : Cotation de l'état de conservation des os coxaux inspiré de l'ICA [13]

Table 1 : Scores of preservation of os coxae inspired by the IPA [13]

Tableau 2 : Stades de cotation des sous-caractères de la méthode de Bruzek [2]

Table 2 : Scoring sexualization based on five characters of the method elaborated by Bruzek

Tableau 3 : Répartition en pourcentage des individus non observables en fonction du sexe par côté

Table 3 : Percentage distribution of unobservable individuals according to sex by side

Tableau 4: Concordance des diagnoses sexuelles entre le terrain et le laboratoire

Table 4: Sexual diagnosis concordance between field and laboratory

Figure 1 : Stade de conservation de l'os coxal (DAO : Cindy Dentz)

Figure 1 : Preservation stages of os coxae (Computer Aided Drawing : Cindy Dentz)

Figure 2 : Répartition des 8 stades de conservation des os coxaux de notre corpus $(\mathrm{N}=602)(0=$ absent, $1=$ non identifiable, $2=0 \%<$ ICA $<25 \%, 3=25 \% \leq$ ICA $<50 \%, 4=50 \%, 5=50 \%<$ ICA $<75 \%, 6=75 \% \leq$ ICA $<100 \%, 7=100 \%$ )

Figure 2 : Distribution of the 8 stages of preservation of the os coxae from our corpus ( $\mathrm{N}=602)$ $(0=$ absent, $1=$ unidentifiable, $2=0 \%<$ ICA $<25 \%, 3=25 \% \leq$ ICA $<50 \%, 4=50 \%, 5=50 \%<$ ICA $<75 \%, 6=75 \% \leq \mathrm{ICA}<100 \%, 7=100 \%)$

Figure 3 : Attribution en pourcentage d'une forme sexuelle par critère anatomique $(\mathrm{N}=525)$.

Figure 3 : Percentage attribution of a sexual form by anatomical criteria $(\mathrm{N}=525)$

Figure 4: Effectif des mesures de la DSP [1] : PUM Longueur acetabulo-symphysaire, SPU largeur cotylo-pubienne, DCOX longueur de l'os coxal, IIMT hauteur de l'échancrure ischiatique, SCOX largeur de l'ilium, SS longueur spino-sciatique, SA longueur spinoauriculaire, SIS largeneur cotylo-sciatique, VEAC diamètre acétabulaire verticale.

Figure 4: number of implemented measurements according to DSP [1]: PUM acetabulosympyseal pubic length, SPU cotylo-pubic width, DCOX coxal length, IIMT greater sciatic notch height, SCOX iliac breadth, SS spino-sicatic length, SA spino-auricular length, SIS cotylo-sciatic breadth, VEAC vertical acetabular diameter.

Figure 5 : Fréquence des 8 stades de conservation de l'os coxal $(0=$ absent, $1=$ non identifiable, $2=$ $0 \%<\mathrm{ICA}<25 \%, 3=25 \% \leq \mathrm{ICA}<50 \%, 4=50 \%, 5=50 \%<\mathrm{ICA}<75 \%, 6=75 \% \leq \mathrm{ICA}<$ $100 \%, 7=100 \%)$ selon la morphologie masculine ou féminine, par individu ( $\mathrm{N}=302)$.

Figure 5 : Frequency of the 8 stage of os coxae preservation ( $0=$ absent, $1=$ unidentifiable, $2=0 \%$ $<$ ICA $<25 \%, 3=25 \% \leq \mathrm{ICA}<50 \%, 4=50 \%, 5=50 \%<\mathrm{ICA}<75 \%, 6=75 \% \leq \mathrm{ICA}<100$ $\%, 7=100 \%)$ according to male or female morphology, per individual $(\mathrm{N}=302)$.

Figure 6: Pourcentage des mesures de la DSP qui ont pu être prises en fonction de la morphologie masculine ou féminine et valeur du p du test de Fisher. 
Figure 6: Percentage of DSP measurements that could be taken based on male or female morphology and Fisher's test p-value 